pletely destroyed. When the hepatic vessels are affected by inflammatory disease transmitted from the portal, their walls are thickened, the lumen greatly increased, and the vessels are filled with thrombi in different stages of disintegration, or abundance of pus corpuscles, which being mingled with the blood of the inferior vena cava, are poured into the right side of the heart, from thence to the lungs, where they may give rise to infarctions, embolic abscess, or true embolic pneumonia.

The opportunity to examine a more unique case than the present is rarely presented. The microscopic examination of various organs afforded an admirable opportunity to verify the teachings of pathology, and observe many of the intermediate steps in the development of the fatal disease.

Sections from the liver in the vicinity of the abscess and through the abscess wall showed the liver cells unaffected but greatly distorted, from pressure, so much so as to appear like columnar epithelium; the walls of the portal and hepatic veins were greatly thickened, and the calibre of the latter much increased, while both systems of vessels were filled with granular detritus, pus corpuscles, and thrombi in various stages of disintegration. Sections from the affected portions of the lung showed the alveoli filled with granular matter, while the vessels containing the emboli were apparently unaffected. Thrombi were found in the vessels leading to the affected portions, but without the radius of the diseased area. Examination of the kidney showed casts in abundance within the uriniferous tubules, but few perceptible changes in the cortex or interstitial tissue.

The case seems, from the pathological conditions observed, to have been one of phlebitic rather than of pyæmic origin. It is of interest to determine the exact spot where inflammation originated, whether at the point where the pin was found or within the liver. It certainly would seem to be a just conclusion that it arose at the point of greatest mechanical irritation, where thrombi were formed from pressure exerted by the swollen mucous membrane, and later rendered septic by the absorption of pus. Yet it certainly would seem reasonable to suppose that the parts about the appendix would have shown greater evidence of inflammation. The disturbance in the kidneys, doubtless, was of an irritative character, from the passage of the biliary salts producing congestion.

The case is particularly instructive in its clinical features, as showing how grave and fatal a disease may exist within the liver and other organs unattended by pain or soreness, the two earliest symptoms thought to be of greatest diagnostic value. Also as showing how a purely local irritative cause may originate disease of a benignant character which later may become malignant. It is noticeable that in the present case there were no inflammatory processes in the neighborhood of the crecum which could occasion any severe symptoms during life, hence differing from the class of cases described as typhlitis or perityphlitis; that there was no perforation or peritonitis; that in the absence of local symptoms the diagnosis could not at the best have reached a greater precision than to infer the existence of some pyæmic or phlebitic process from some internal cause. It is therefore probable that other cases described as pyæmic of internal origin, and unconfirmed by post-mortem and microscopic examination, may have a similar origin.
N. B. It was not known that the child had swallowed a pin until it was shown to the parents after autopsy, when her sister said she remembered when it bappened, but had quite forgotten the occurrence. According to the latter's statement the pin was swallowed about the middle of August, and the fatal illness began November 10, 1882.

\section{A PHYSIOLOGICAL METHOD OF TREATING CARIES OF THE DORSAL VERTEBRA.}

BY Charles f. Strllman, M. S., M. D., NEW YORK, Orthopadic Surgeon to the New York Infant Asylum.

Carres of the vertebræ is recognized as occurring most frequently in the dorsal region, ${ }^{1}$ and it is also considered the most difficult to cure in this region ${ }^{2}$ by any of the methods in vogue.

If simple fixation braces be used, such as Davis's, Washburn's, Knight's, or Shaffer's, there is not sufficient backward traction produced to relieve the diseased bodies of the vertebre from weight, nor is there forward pressure produced about the seat of the disease to combat deformity.

If symmetrical extension is desired, and the plaster jacket is used for this purpose, the jury mast will be required in addition, if the disease be in the middle or upper dorsal region, and the apparatus is cumbersome and not adjustable.

Wyeth's or Roberts' local extension jackets are not available unless provided with a head rest, and are then too heavy and unmanageable for nice practice. ${ }^{3}$

Backward traction is the most convenient and useful plan of treatment which we have at command. This aims to curve the spine backward so as to relieve the bodies of the vertebræ from the superincumbent weight which is thereby transferred to the posterior processes.

There are several methods of effecting this : the use of the plaster jacket applied while the patient lies face downward in a hammock (Dary), or upon two bands, - pelvic and thoracic-(Halsted), constituting one; the fixation brace of Mr. E. J. Chance, ${ }^{4}$ of London, which is provided with pads opposite the seat of disease, from which the upper part of the metal frame is bent backward at an angle, being a second method; while the use of the double knuckle joint lever brace of Dr. C. F. Taylor, ${ }^{5}$ of New York, constitutes a third. The latter not only curves the spine backward, but by its forward leverage upon the transverse processes of the diseased vertebræ tends to reduce or prevent the deformity.

It is, however, very heavy, since its peculiar construction necessitates great strength of material, as it is so arranged as to bring an unnecessarily severe pressure upon the spine at the seat of disease, leading in some cases to excoriation. ${ }^{6}$ These systems of treatment have, with few exceptions, been before the profession for a sufficiently long period, and have been employed with varying degrees of success; but, on the

1 Pott's Disease. By Newton M. Shaffer, M. D. G. P. Putnam's Sons. 1879. Page 45 .

2 Ibid., page 51 .

8 For an illustrated description of these various methods see Amer ican Journal of Obstetrics, June, July, August, and September, 1883. 4 'The Surgery of Deformities. By E. Noble Smith. London. Smith, Elder \& Co. 1882. Pages 224-226.

5 Transactions of the New York State Medical Society, 1863.

6 Orthopædic Surgery and Diseases of Joints. By Lewis A. Sayre. New York. D. Appleton $\&$ Co. 1883. Page 183 . 
whole, the mechanical treatment of Pott's disease is considered to be in an unsettled and unsatisfactory condition.

In the consideration of the mechanical problem presented by this disease there are two requisites which must enter for successful treatment:-

(1.) Arrest of the disease.

(2.) Improvement of the deformity.

And as a result of research in this direction a new form of lever brace is proposed which is adapted to meet these requirements.

Its objects are (1) to produce extension of the bodies of the vertebræ by backward traction, thus aiming at the arrest of the disease; and (2) to exert forward pressure at the seat of deformity, thus tending to its improvement or obliteration. These objects are also embraced by the instrument of Dr. Taylor, but in the brace to be described they are attained by a different construction aud by a more ecouomical application of forces which entails special advantages.

To properly understand the principle upon which the new brace is constructed we take a patient with Pott's disease, having a well-defined knuckle, and lay him upon the back on a table, the edge of which comes to the apex of its deformity, as in Fig. 1, his

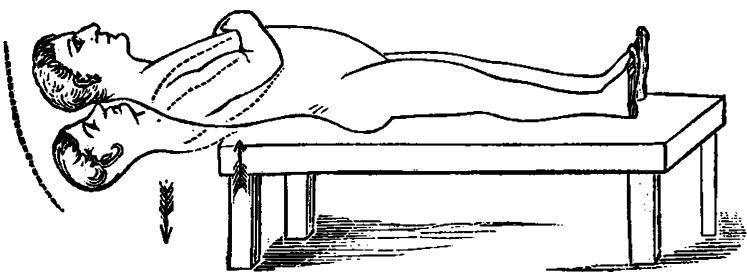

F1G. 1. Extension of bodies of vertebræ by backward traction.

head and shoulders being allowed to fall downward. This is effected by the law of gravitation, and the weight of the part of the body above the site of the disease is thus utilized to produce an extension of the bodies of the spine at the knuckle. The edge of the table (which, of course, should be so padded as to bring the pressure upon the transverse, and not the spinous, processes) presses upon the deformity to such an extent as to improve the form of the knuckle, and this partially or entirely disappears until the erect position is again assumed, unless the consolidation process has proceeded to such an extent as to render effort in this direction futile. See Fig. 1 .

We have thus by this position produced the two mechanical effects upon the spine which we have already stated were necessary to the successful treatment of the disease, and if this position could be kept up indefinitely there would be a rapid improvement; but it is not feasible to keep the patient in this position, and we are therefore led to embody in a brace the forces which will produce and sustain this condition of the spine in whatever position the body may assume.

To do this we construct the brace in two parts, one to represent the table and the other the backward traction power, - which in the position just shown is exerted by the attraction of gravitation.

The table part of the brace consists of a firm pelvic band, from which strong padded strips pass upward on either side of the median line to the seat of disease. See Fig. 2. The backward traction part consists of a back frane, secured on the pelvic girth by a rachet, which admits of its adjustment at any angle with the table part of the brace, and thus regulates the degree of traction power, which may by it be varied from a simple upright support to a powerful lever, depending only on the angle at which this back frame is thrown out and secured. (Fig. 3.) The upper part of this

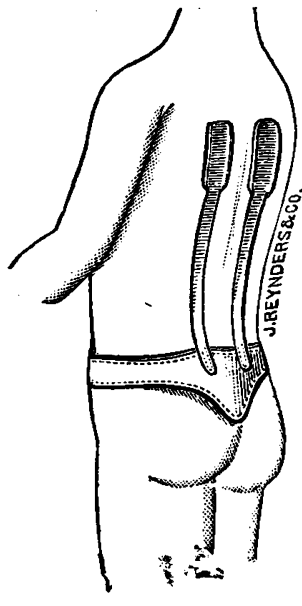

FIg. 2.

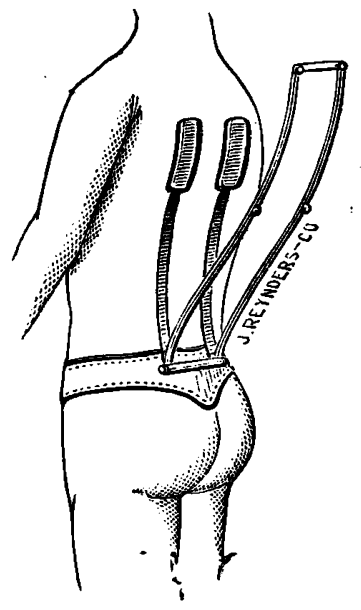

Fro 3 backward traction frame is secured to the body by padded straps connected to a chest plate in front, to avoid constriction, and when bound down to the body, as in Fig. 4, presents the appearance there shown.

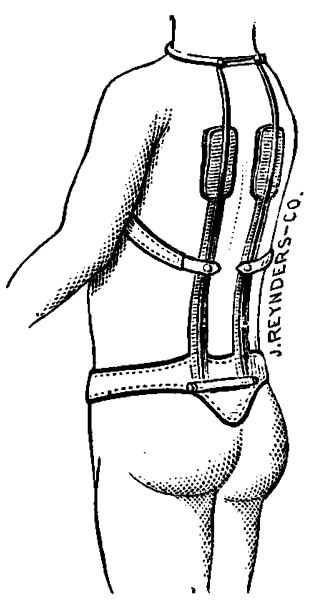

Fig. 4.

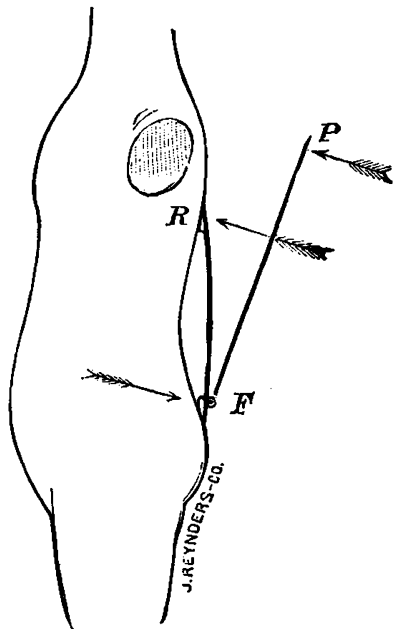

Fig. 5.
We thus perceive that by means of the two parts of this brace we can obtain the attributes desired in an effective dorsal apparatus, its mechanical action being shown in Fig. 5, by which it will be seen that after the traction frame, $F \mathbf{P}$, is securely fastened at $F$ at an angle with the table frame, $F$, and the whole firmly fastened to the body, the forward tendency of the part of the body above the seat of disease would be combatted by the pads at $R$, and this forward tendency would at the same time act to force in the knuckle by pressure over the transverse processes of the diseased vertebræ, so that we would have a corrective automatic effect produced by the brace to keep the body erect, and at the same time improve the deformity.

Also that the spring effect produced by the setting off of the traction frame and drawing the body backward against it would cause the brace to be held more tightly against the back, and also produce the highest degree of fixation of the spine of which any brace is 
capable. In the beginning of the treatment in a severe case, it is well to have the traction frame set off at such an angle as to cause considerable pressure upon the sides of the knuckle; but this angle may be lessened week by week as the patient improves, until finally the traction frame lies directly upon the pad;, and becomes a mere fixation brace or support without any leverage whatever. If the case be slight or of recent date, the angle of the traction frame need be but a slight one; and as the instrument is an anteroposterior brace in the truest sense, it may be included in the commendatory remarks of Professor Shaffer, ${ }^{1}$ who summarizes among the principal advantages of the antero-posterior support : -

"(1.) The ease with which it can be adjusted and the great comfort experienced by patients who wear it.

"(2.) It can be removed with safety at any time by placing the patient in the prone position, when such modifications can be made as are necessary to the comfort of the patient or the treatment of the case.

"(3.) The concentration of the requisite pressure at suitable and convenient points without interfering with transpiration or respiration; and, finally, the cleanliness and lightness of the whole apparatus, matters which certainly ought to be consulted in a long and necessarily tedious treatment."

\section{POISONOUS MUSHROOMS.2}

by JUlius a. Palmer, Jr.

Frequent inquiry as to the best text-book on the subject of esculent and poisonous fungi leads us to notice here the two works whose titles are given in the note.

'The work of Cordier is by far the most complete and of the most practical use of any yet published; while the more recent contribution to this branch of science made by Sicard contains evidence of the most patient research on the subject of the reproduction of fungi, a problem difficult of solution even by those best acquainted with this branch of the kingdom of nature.

Both attempt to pronounce upon the qualities of each species, and in that they differ from any current English work. The labors of Cooke and Berkeley are chiefly devoted to a proper classification, while those of Badham, Smith, and Hussey are too limited in their scope to be considered as text-books.

In January, 1879, the present writer contributed to the Moniteur Scientifique, of Paris, an article on the peculiar poison of the Amanita family, which article was copied and commented upon by some American medical journals. Nothing in either of these works would controvert its conclusions, which may be briefly restated as follows: Mushrooms are unfit for food by decay or other cause, producing simply a disagreement with the system, by containing some bitter, acrid, or slimy element, or by the presence of a wonderful and dangerous alkaloid, which is absorbed in the intestinal canal. This alkaloid, so far as known, is found only in the Amanita family, and auy child can learn to rec-

1 Pott's Disease. Shaffer. 1879. Pages 47, 48.

2 Les Champignons. Histoire, Description, Culture, Usages, etc. Par F.S. Cordier. Orné de Vignettes et de 60 Chromolithographies. Paris: J. Rothschild, Elliteur. 1876.

Histoire Naturelle des Champignons Comestibles et Vénéneux. Par G. Sicard, ouvrage accompagné de 75 Planches Coloriées. Paris: Libraire Ch. Delagrave. 1883. ognize and reject this whole family by certain distinguishing marks, the most important of which is the volva or cup at the base of the stem.

Several attempts have been made, and not very successfully, to isolate this alkaloid; among these may be meutioned that of Schmidberg and Koppe, who obtained a colorless alkaloid to which they gave the name of muscarine; of Boudier, who, using the Amanita bulbosa, gave to his extract the name of bulbosine.

Mr. Sicard gives the following comparatively recent and additional testimony to the presence of the same alkaloid in another member of the Amanita family :-

“In 1868 Doctors Curie and Vigier sought to isolate the alkaloid of poisonous mushrooms. They used exclusively the Amanita mappa, because this seemed to them the most poisonous. Dr. Vigier has given to this Amanita two pharmaceutical forms: First, an alcoholic extract prepared by leaving for a fortnight in contact one kilogramme of fresh poisonous mushrooms crushed and one kilogramme of alcohol ninety per cent. proof. The mixture is then pressed and filtered. This tincture is given in doses of four to six grammes a day in certain catarrbal affections. Second, in the form of pills of five centigrammes of extract resulting from the distillation of the alcoholic mixture. These pills are taken in doses of three or four a day. Dr. Curie will soon publish the results of his therapeutic researches on this subject."

The use of this alkaloid as a remedial agent has long been considered favorably, and has, perhaps, made little progress chiefly on account of the difficulty of identification of species, since to the novice all toadstools look alike.

The Amanita muscarius (common in New England) has also been successfully employed in the treatment of chronic catarrh, profuse perspiration, skin diseases, and epilepsy.

Dr. Curtis, of South Carolina, has proposed to use the Amanita phalloïdes as a specific for cholera, Bright's disease, and intermittent fevers. His experiments were not sufficiently extended to be conclusive, but Mr. Cordier considers them worthy of repetition, and adds: "Why should not the narcotic properties of this mushroom render it a substitute for opium? It would have an advantage in the matter of its cost, for it asks for no culture. There are few wooded districts where it is not found abundantly."

There are other fungi whose peculiar properties have made them active medicinal agents so far as tested; among these the acrid milk mushrooms have been prescribed with success in phthisis, oven in its more advanced stages.

But it may be noted that the authorities cited above, to which I would add my modest testimony, all recognize the Amanita alkaloid as the best defined of any of the toxic properties of the fungi. All these varieties are common to our woods at certain periods of the year after heavy rains. If any member of the medical profession should wish to test their properties, I should be only too happy to identify them for his experiments.

While recognizing the great value of both these contributions to a branch of science yet imperfectly understood, I regret to note very little progress in defining the action of the Amanita alkaloid, or in the more iniportant matter of the treatment of a case of poisoning by such. Both of these writers advise only means 